\title{
Factors Contributing to Pregnancies Among Tertiary Students at the University of Namibia
}

\author{
Ben Victoria ${ }^{1}$, Daniel Opotamutale Ashipala ${ }^{1} \&$ Nestor Tomas ${ }^{1}$ \\ ${ }^{1}$ Department of General Nursing Science, School of Nursing, Faculty of Health Sciences, University of Namibia \\ (UNAM), Rundu, Namibia \\ Correspondence: Daniel Opotamutale Ashipala, Department of General Nursing Science, School of Nursing, \\ Faculty of Health Sciences, University of Namibia (UNAM), PO Box 88, Kaisosi Road, Rundu, Namibia. Tel: \\ 264-66-268-6009. E-mail: dashipala@unam.na
}

Received: February 26, 2020 Accepted: November 10, 2020 Online Published: November 20, 2020

doi:10.5539/gjhs.v13n1p36 URL: https://doi.org/10.5539/gjhs.v13n1p36

\begin{abstract}
The Government of Republic of Namibia through the services rendered by the Ministry of Health and Social Services (MoHSS) continues to provide various free health services including contraceptives to its citizens. Nevertheless, several challenges related to unplanned pregnancies among tertiary student's remains a challenge, which includes poor reproductive health status, socio-economic consequences rapid-population growth, rural-urban migration of youths accompanied by proliferation of informal settlements around cities, high youth unemployment and crime. This requires institutions of higher learning to establish which strategies are likely to address these problems of unplanned pregnancies among tertiary students. The aim of this study was to explore and describe the contributing factors to pregnancies amongst tertiary students at a selected satellite campus in order to make recommendations to the University of Namibia (UNAM).

An exploratory, descriptive and qualitative design was used. The study was contextual in nature. A convenient sampling was used. The data were collected through three focus-group discussions with 19 students from the three faculties namely: Education, Health Sciences and Management Sciences. Data were analyzed through qualitative content analysis. Strategies to ensure trustworthiness and ethical implementation of the study were implemented. It became evident from the study findings that factors which are contributing to pregnancies, as evidenced by the four emerged themes namely: Personal factors, institutional related factors and improvements measures. This study has implications for higher education institutions in terms of promoting sexual and reproductive health information and increasing access to a range of contraceptive methods of campus which are key in the prevention of pregnancies among tertiary students. Participants in this study recommended that peer educators and students counsellor within the campus should be used as a vehicle to provide support and guidance to students on reproductive health choices.
\end{abstract}

Keywords: challenges, contraception, factors, pregnancy, student

\section{Introduction}

Worldwide, every year, around 87 million women become pregnant unintentionally, and this is a significant number, (WHO, 2018). According to Bearak, Popinchalk, Alkema, and Sedgh, (2018), during the period between 2010 and 2014, an estimated $44 \%$ of pregnancies worldwide were unintended. This translates to a rate of 62 unplanned pregnancies per 1,000 women aged 15 to 44 years old Bearak et al. (2018). There was also a significant difference between overall unintended pregnancy rates in developing and developed regions. In the same period, there were 65 unintended pregnancies per 1,000 women aged 15 to 44 in developing regions, compared with 45 per 1,000 women in developed regions. The high rate of unintended pregnancy in developing regions is said to be related to the great unmet need for contraception in those areas, Bearak et al. (2018).

Additionally, in low and middle-income countries, an estimated 16 million young women aged 15 to 19 , and about a million girls under 15 years of age give birth every year (Dick \& Ferguson, 2015). Moreover, Adolescent child bearing (intended or not) has adverse effects at the individual, community, and societal level. Compared with their peers who delay childbearing, young women who have babies are less likely to finish high school, more likely to be poor as adults, and more liable to have kids who have poorer behavioural, educational, and health outcomes over the course of their lives (Cook \& Cameron, 2015). 
Statistics for South Africa for 2017 reported that prevalence of pregnancy increased with age, rising from $0,6 \%$ for females aged 14 years, to 10, 7\% for females aged 19 years (General Household Survey, 2017). Most students at higher institutions are actively involved in searching and discovering, as well as sexual experimentation. For this reason, students very so often engage in unsafe sex, which put them at a higher risk of contracting STIs, (including HIV/AIDS,) as well as unwanted pregnancies. In addition, unwanted pregnancy is the main cause for female students dropping out from school and poses serious health, social, and economic problems for student. At the same time, it reported that adolescent pregnancy is a major cause of interrupted and discontinue education (Fekede, 2015).

WHO, (2018) of the estimated 211 million pregnancies that occur each year, about 46 million end in induced abortion. The rate of adolescent pregnancies in the Kavango region is $27 \%$ of all adolescent pregnancies in Namibia, and this is the highest rate nationwide. Moreover, Kavango region has the lowest average age at first birth in Namibia, which is 19.3 years old (NDHS, 2013). The government of Namibia has a great policy in place, of providing different types of contraception free of charge, so as to prevent unintended pregnancies, (Consolidated National Reproductive and Child Health Policy, 2008). However, according to UNICEF, in Namibia, $76 \%$ of sexually active adolescent girls (aged 15 to 19 years) do not use modern methods of contraception, (UNICEF, 2013). Statistics also reveal that $6.8 \%$ of adolescent girls aged 15 to 19 have their first sexual encounter by the age of 15 , and an additional $3.9 \%$ of women, by age of 20 to 24 years old. This therefore means that at the time of enrolling into universities, students are at an age of about four years above the popular age of first sexual encounter in Namibia, suggesting that they are usually sexually active, (UNICEF, 2013).

Adolescence is widely known as a developmental phase in the human lifecycle between childhood and maturity and a lot of changes take place which affects individuals in different ways (Thongnopakuin, Pumpaibool, \& Somrongthong, 2018). During this phase young people go through different changes in terms of their physical, cognitive, emotional, and sexual development. These changes turn out to be risk factors for premarital sexual behaviour, adolescent pregnancy, abortion, and sexually transmitted diseases (STDs), such as acquired immunodeficiency syndrome.

The practices of university students with regards to contraception are reported to be low in Namibia (Tatenda, Ashipala, Endjala, \& Tomas, 2019). However, throughout the world, female students are at risk of unplanned pregnancies as a result of ineffective use and non-use of contraceptives, Coetzee and Ngunyulu, (2015). Consequently, pregnancy among tertiary students is one of the major cause of interrupted and discontinue education (Fekede, 2015). Pregnancy has a detrimental effect on the education and future of tertiary students, this is because young mothers are obliged to attend to their education irregularly and sometimes drop out of school. Cultural practices is identified as one the factor to pregnancies among students as it prohibit young women to talk about sex at home with their mothers nor with anybody else (Krugua, Mevissena, Münkela, \& Ruitera, 2018). Absence of designed programs that teach students regarding sex education at the campus was reported as a major contributing factor to pregnancies among tertiary students (Fekede, 2015). Additionally, female students who used alcohol and drug abuse were more likely to experience teenage pregnancy compared to those who did not use (Mathewos \& Mekuna, 2018).

\section{Methods}

\subsection{Design and Setting}

The study used a qualitative research design, in particular, an explorative, descriptive and contextual research design to explore the factors contributing to pregnancies among tertiary students as described by them individually (Babbie \& Mouton, 2016; Creswell, 2013). The study was conducted at the University of Namibia, north-east region of Namibia. The satellite campus is part of the University of Namibia and offers undergraduates courses through three faculties namely: Education, Health Sciences and Economic and Management Sciences. Qualitative research is concerned with the everyday human experiences within a natural environment (Maree, 2016). The focus group discussions which were conducted enabled the researcher to search for similarities in the meaning of the core factors that are deemed to be the root contributing factors of pregnancies among participants. The study focus group discussion focused on exploring the contributing factors of pregnancies among students as they have had occurred (Creswell, 2013,). The use of the used focus group interview was facilitated through the use of open ended questions which oriented the participants to the study (De Vos, Strydom, Fouche, \& Delport, 2011).

\subsection{Population and Sampling Strategy}

Grove, Burns, and Gray (2013) define a study population as a bigger pool from which sample elements are 
drawn. The population in this study consisted of 804 students enrolled at a University of Namibia, in three faculties, namely: Health Sciences, Education and Economic and Management Sciences. A sample is a subset of the population elements (Polit \& Beck, 2017). The sample in this study comprised of all students studying at University of Namibia in 2019 on a fulltime study mode. The researchers approached participants face-to-face in the classroom at the university campus and the purpose and objectives of the study were explained to them. A convenient sampling was used to drew a sample of nineteen $(\mathrm{n}=19)$ readily available students from a total of 804 registered at the campus (Brink, van der Walt, \& van Rensburg, 2018).

\subsection{Data Collection Methods}

Grove et al. (2013,) define data collection as the exact, systematic gathering of information related to the research purpose of the intended study. Data in this study were collected between 10 July 2018 and 28 October 2018, through focus-group discussions. The students that were willing to participate in the study were recruited by the researchers, while the time and venue for discussions were arranged with them accordingly. A total of three focus-group discussions were conducted, which consisted of each six, six and seven participants each. The discussions lasted about 50 to 60 minutes, and the number of discussions held was determined by data saturation (Polit \& Beck, 2017). The researcher used a semi-structured focus-group discussion guide, with a prepared written topic guide prior to the discussions; however, probing questions were used to further explore participants' responses and to stimulate more detailed information (Polit \& Beck, 2017). All focus-group discussions were audio recorded using a digital voice recorder. Pilot testing was conducted with a focus-group discussion of 6 students, which helps to refine the topic guide and other data-collection plans (Creswell, 2013).

\subsection{Analysis}

The aim of the data analysis process is to organise, impose structure on and elicit meaning from data which has been collected (Groove, 2013). In this study content analysis was used to analyse the data as this is deemed to be the most reliable method used in qualitative research as it is fairly systematic and it allows the researcher to organise the information into themes and sub-themes (Leedy \& Ormrod, 2013). The transcribed interviews and narratives from the research notes were organised into codes, main themes and sub-themes. An independent coder then verified the accuracy of the analysed data and held a meeting with the researcher to discuss and agree on the themes which had been identified. Data were stored in a computer encrypted with a password and restrict access to this document was only allowing primary investigators access.

\subsection{Data Trustworthiness}

Furthermore, trustworthiness was ensured following the principles of Lincoln and Guba (1985), which consists of the criteria of credibility, dependability, conformability and transferability.Credibility was ensured through prolonged and varied engagement with the participants in the field while objectivity was maintained throughout the data collection process. Testing of the interview guide was conducted with three students who were not part of the study. However, no major changes were made to the focus group interview guide questions after the testing of the focus group interview guide. In order to ensure both confirmability and dependability an experienced independent coder analysed the data. The final themes and subthemes were identified after a consensus has been reached during a meeting between the researcher and the independent coder. A chain of evidence was kept through the detailed recording of both the research methodology and the data analysis process.

\subsection{Ethical Considerations}

Ethical approval to conduct the study was obtained from the School of Nursing Research Committee at the University Of Namibia Faculty of Health Sciences. The researcher obtained the participants' personal consent in relation to their participation in the study after the purpose and significance of the study has been explained to them. Ethical principles were adhered to throughout the study in order to protect the rights, dignity and safety of the participants (Grove et al., 2013). Participation in the study was voluntary and the participants were free to withdraw from the interviews at any time if they so wished (Creswell, 2013). Confidentiality was ensured as each participant was given a numerical code at the beginning of the structured interviews which was then used to refer to the participant during the interviews and which subsequently appeared on the transcript of the interview to ensure anonymity (Grove et al., 2013).

\section{Findings}

\subsection{Description of Study Participants}

The participants were all fulltime undergraduate students studying at the University of Namibia, Rundu campus 
situated north-east of Namibia. All the participants were under the age of 40. The majority of the participants were unemployed. The characteristic of study participants is given in the Table 1 below.

Table 1. Characteristics of participants

\begin{tabular}{ll}
\hline Age & 15 \\
\hline $18-30$ & 4 \\
$31-40$ & 0 \\
$41-50$ & 8 \\
\hline Ethnic tribe & 5 \\
\hline Oshiwambo & 3 \\
Kwangali & 3 \\
Nyemba & \\
Others & 0 \\
\hline Gender & 19 \\
\hline Male & 17 \\
Female & 2 \\
\hline Marital status & 6 \\
\hline Single & 6 \\
Married & 6 \\
\hline Faculty & 7 \\
\hline Health Sciences & \\
Economic and Management Sciences & 3 \\
Education & 4 \\
\hline Level of study & 6 \\
\hline First year & 7 \\
Second year & \\
Third year & \\
\hline Fourth year & \\
\hline
\end{tabular}

The themes and subthemes from the data analysis are indicated in Table 2 below.

Table 2. Themes and Sub-themes which emerged from the data analysis.

\begin{tabular}{ll}
\hline Themes & Sub-themes \\
\hline & 3.2 .1 Socio - economic factors \\
& 3.2 .2 Lack of sexual and reproductive health information \\
& 3.2 .3 Cultural factors \\
& 3.2 .4 Peer influence \\
3.2 Personal factors & 3.2.5 Alcohol and drug abuse \\
& 3.2.6 Gender power relation \\
\hline \multirow{3}{*}{ 3.3 Institutional factors } & 3.3 .1 Lack of recreational facilities \\
& 3.3 .2 Lack of contraceptive on campus \\
\hline & 3.4 .1 Access to the NSFAF funding \\
3.4 Mechanism for improvement & 3.4.2 Sexual awareness programmes and campaigns \\
& 3.4.4 Peer educators/counsellors within the campus \\
\hline
\end{tabular}




\subsection{Theme 1: Personal Factors}

This theme reflects the factors that are perceived to be contributing to pregnancies among tertiary students at the University of Namibia. The sub themes expressed by participants include socio-economic status, lack of sexual and reproductive health information, cultural factors and peer influence.

\subsubsection{Socio-Economic Status}

Participants revealed that students are dating of older men in the society who are able to render financial support to them. This was confirmed by few extracts which are provided below:

"It's a personal decision whether to date a man with money so you can buy yourself an iphone and other fancy stuff)" (P2G1)

Some participants who indicated to come from poor families confirmed that being at the university require smart phones as the university prefer to communicate through students' portal and most of the academic activities require the use of smart phones. Their family cannot afford to buy them one while dating a man you love, who can afford can be alternative.

"Just make a wise decision that fit your circumstance, but of course the problem if he wants a family it will mean I may sacrifice to get pregnant as his reason is that he can afford to take care of the baby" (PlG3).

\subsubsection{Lack of Sexual and Reproductive Health Information}

The storylines provided below attest that participants were worried by the fact that there is no sexual and reproductive health information on campus.

"Imagine you are first year, fresh from secondary school, it is difficult to make the right options with regard to sexual and reproductive health" (P6G2).

"There is no facilities on campus and public facilities have long queues, one must miss classes the whole day to access sexual and reproductive health information" (P5G3).

One participant indicated her disappointment on the unavailability of Awareness Clubs on campus to educate and sensitise students on sexual and reproductive health.

"I have never got any sexual information on the campus, maybe now that the school of nursing is her, things will improve" (P7G1).

\subsubsection{Cultural Factors}

Most participants acknowledged that parents are reluctant to discuss sex related issues even with grown up children. Most participants indicated that some culture expect women to give birth or even get married early in life. The expressions from the participants regarding culture presented below clarified how they felt:

"My parent would never talk to me about sex" (P3G1).

On the other hand, one participant who confirmed talking to her mother about sex was informed that it was a taboo to discuss sex with children.

"As I tried to talk to my mother about sex, she immediately told me to shut up" (P7G2).

\subsubsection{Peer Influence}

It was evident in most participants' responses that friends influence each other's' decisions about sex. Most participants acknowledged that they had discussed sex with their friends and that sometimes had to indulge into unprotected sex because a friend promoted it them.

"I was moved by my friend' statement...Sex is a basic need and having a child while younger have more benefits" (P6G3).

"If you cannot give your man a child, while having sex with him, you are regarded as a prostitute" (P5G1).

\subsubsection{Risky Sexual Behaviours}

Some participants believed that most of the students abuse alcohol and drugs end up indulging in unprotected sex.

“...You know how it feels to be tipsy... you may be less worried of the consequences and your guy may take advantage" (P4G1).

Most participants confirmed the abuse of alcohol by indicating that they spent most of their free time at bars and shebeens drinking alcohol. 
"One get excited when it is a Friday, we contribute for booze" (P4G2).

One participant confirmed that some students especially man take drugs to give them extra power in bed.

"While drug may give him power to satisfy you in bed, he may not be himself in making decisions on the usage of condom” (P1G3).

\subsubsection{Lack of Contraceptive Use}

Most of the participants indicated that some students are just ignorant on the use of contraceptives.

"Sex emotions are too strong to look for a condom" (P5G1).

Some participants indicated that women now use the safe period to indulge in sexual activities and they no longer depend on the use of contraceptives.

"I often have sex immediately after my periods are over" (P5G2).

\subsubsection{Gender Power Relation}

Most participants acknowledge having placed men a superior social status that allows them to make decisions on sexual matters.

"If a man decides on having sex, you cannot stop him" (P4G3).

Some participants expressed that it is socially acceptable and sometimes encouraged for men to be involved in premarital sexual activities while women must be passive and sexually innocent.

"If a man does not care, he likely not to use condom... as a women you have little control" (P5G1).

\subsection{Theme Three: Institutional Related Factors}

This theme reflects the responses given by participants when described how institutional challenges contributes to pregnancies among students on campus. The sub-themes emerges out of this theme includes, lack of recreational facilities and lack of contraceptives on campus.

\subsubsection{Lack of Recreational Facilities/Boredom}

Most participants expressed that there are no enough recreational facilities on campus and this may force many students to indulge in unproductive activities including sexual activities.

"You hardly have sport games on campus" (P3G3).

"The gym facilities on campus are not utilised... gymming would kill my boredom" (P4G1).

\subsubsection{Lack of Contraceptives on Campus}

Most of the participants indicated that condoms are not readily available in the toilets. Both men and women would at least take if condoms are placed in places of convenience.

"I would go to the nearest bar if I need condoms" (P1G3).

"There is no clinic to provide reproductive health services on campus" (P1G2).

Some participants who reside in the hostel however indicated that condoms are available at the office of the student counsellor but, only placed at one place, making it difficult for every to feel comfortable to go there when others are watching.

"I feel embarrass to enter that office and request condoms when others can see me" (P1G2).

"Condoms are not being placed at the places of convenience" (P6G3).

\subsection{Theme Three: Mechanisms for Improvement}

This theme emanated from participants responses when asked to express their opinion on what can be done to improve the current situation with regard to high rate of pregnancy on campus. The suggestions include access to Namibia Students' Financial Assistance Fund (NSFAF) funding, sexual awareness programmes and campaigns, accessibility of contraceptives and having peer educators on campus.

\subsubsection{Access to NSFAF Funding}

When asked about what can be done to reduce high rate of pregnancy among student on campus, most participants yearned for access to NSFAF funding to address students' economic hardship.

"If only NSFAF can settle student's accounts and pay out non tuition fees on regularly basis" (P6G3).

"NSFAF system is ineffective and frustrating...I did not get my refund for last year" (P7G2). 


\subsubsection{Sexual Awareness Programmes and Campaigns}

Participants in this study revealed that Student Representative Council (SRC) under the office of Dean of students encourage the establishment of Awareness Clubs on campus to sensitize and conduct sexual awareness campaigns.

"Let the SRC of Gender Affairs and Community development take the issues of sexual and reproductive health seriously" (P2G1).

Some participants' suggested for peer educator programmes to be active on campus.

"We can learn a lot from each other, if peer educators programmes are promoted and supported on campus" (P5G3).

One participant requested for the school of Nursing to be visible and provide sexual awareness campaigns.

"Now that the School of Nursing is here, let them focus on providing sexual awareness on campus" (P7G2).

\subsubsection{Accessibility to Contraceptives}

The study participants suggested that the university provide a site clinic on the campus to cater for students' needs.

"We need to be treated like students at the satellite campus; The University of Namibia must build a clinic on campus for us" (P1G3).

Some participants felt it would be better if the condoms can be placed in all toilets as it is the most used contraceptive.

"Someone must be tasked to ensure that condoms are always available in all campus toilets" (P1G2).

\subsubsection{Peer Educators/Counsellors Within the Campus}

Participants suggested for peer educator to be promoted and supported to be able to conduct peer teaching on sexual and reproductive health.

"Nursing needs to be encouraged to participate in peer education on sexual matters" (P10).

\section{Discussion of Findings}

The purpose of the study was to explore and describe the factors contributing to pregnancies among tertiary students at the University of Namibia. This section, presents discussion of the study in accordance with themes as emerged out of the study namely: Personal factors, Institutional factors and Mechanism for improvement.

\subsection{Personal Factors}

It is reported in this study that some university students from poor background preferred to get romantically involved with men that are working men, as this can enable them to get the needed financial support. The study findings were in line with the Legal Assistance Centre, (2017) report on gender analysis which asserted that ladies were quoted as preferring dating older men who are well off, as a way to gain financial independence.

The study revealed that the unavailability of Awareness clubs on campus. Awareness clubs are key in the distribution and utilization of health related information on sexual and reproductive health. The findings from this study were similar to those of Donatus, Sama, Tsoka-Gwegweni and Cumber, (2018) who indicate that if communication about reproductive and sexual matters is limited it will force the affected persons to rely chiefly on information from peers and boyfriends which often mislead them into risky sexual behaviours. Tladi and Jali (2014) also pointed out to absence of guidance on issues of sex education as one of contributing factor to pregnancy. It was reported in this study that friends have potential positive or negative influences on the decision regarding sex. This best aligns with Tladi and Jali (2014) who found that most early engagement in sexual activities were due to the influence of peers and that peer influence remain on of leading method of learning more about sex. Tatenda et al., (2019) attested that about $46 \%$ of the university students have learned sex through their peers.

Participants in this study reported that parents are not open or are reluctant to discuss sex related issues with their children as it is regarded as taboo in many culture in Namibia. (Krugua, Mevissena, Münkela, \& Ruitera, 2018) also identified cultural practices as one the factor that prohibit young women to talk about sex at home with their mothers nor with anybody else.

Participants in this study reported that most students spent their free time drinking alcohol or taking drugs. Students admitted that being ignorant, being under the influence of alcohol or drugs make them vulnerable to 
poor decision making on the use contraception and led to many getting pregnant (Qolesa, 2017; Conradie, 2019). Fekende, (2015) indicated that about $36 \%$ of unplanned pregnancies comes as result of indulging into sexual activities will under the influence of alcohol. The study revealed that most students know where to get contraceptives but are ignorant to find and use them during sex. The findings of this study concur with the study by Conradie, (2019); Tshomo, Dorji, Wangchuk, and Tshering, (2018); Zungu and Manyisa, (2009) which reaffirmed that most of the pregnancies were as a result of not using or inconsistence use of contraception. Mutinta, Govender, George, and Gow, (2014) echoed that being sexually aroused is a strong biological factor that encourages young people to lose control and engage in sexual acts without using contraceptives and that the use of contraceptive namely condoms is unnatural and hinder skin to skin contact that bring about intimacy experience.

\subsection{Institutional Related Factors}

The study participants pointed out the lack of a campus based clinic as a barrier to sexual health services use among the students. Qolesa, (2017) stated that sexual and reproductive health services are pronounced as organisational factors that influence the number of unplanned pregnancies among students. On the other hand, the study participants pointed out that the lack recreational facility on campus was responsible for student's boredom. Additionally, Qolesa (2017), stressed that boredom may result in inappropriate activities including sex.

\subsection{Mechanisms for Improvement}

Participants in this study acknowledge that an on-site clinic would improve access to sexual reproductive health services. The Legal Assistance Centre, (2017), emphasised that access to contraception prevent unintentional pregnancy and or abortions. Participants dwelled much on the importance of peer educators on campus. The finding lend support to Tatenda et al., (2019) study on knowledge, attitudes and practice of contraception among tertiary students, which revealed that there is lack of peer educators at the University of Namibia, Rundu campus. Tladi \& Jali, (2014) reiterated that awareness campaigns on sexual reproductive health help to reduce unintended pregnancies among students.

\section{Limitations of the Study}

The results obtained in this study were obtained from undergraduate students studying at the University of Namibia, Rundu campus. Therefore, it is not possible to generalize the study results to other satellite campuses of the University. To generalise findings, a survey of a larger target population including their male counterparts who became fathers, should be conducted to assess to which the same findings would be uncovered. Additionally, there were limited literatures on the factors contributing to pregnancies among tertiary students as much of available literature was done on teenage pregnancy.

\section{Implications for the University}

The focus group discussions revealed that the tertiary students were all experiencing challenges related to personal factors, sexual behaviour related factors and institutional factors. Finally, this study highlighted some improvement measures for higher education institution as a way to mitigate the challenges identified in this study.

\section{Recommendations}

Based on the findings of the study the following recommendations were made:

Contraceptives should be made available and accessible to the students on campus. Thus, condoms should be made available in the campus premises to increase accessibility to students. Increase access to the NSFAF funding as a way addressing economic hardships during studies. Peer educators/counsellors within the campus should be used as a vehicle to provide support and guidance to students on life choices. Additionally, implementation of sexual and reproduction health awareness programmes and campaigns within the campus.

\section{Conclusion}

Pregnancies among tertiary students has been recognized as one of the major social problems affecting the tertiary students and it was found that various factors that contributed to the problem were Personal factors, sexual behaviours, Institutional factors, and Improvement measures. This study has implications for higher education institutions in terms of promoting sexual and reproductive health information and increasing access to a range of contraceptive methods of campus which are key in the prevention of pregnancies among tertiary students. 


\section{Acknowledgements}

Researchers thank all the respondents who took the time to participate in this study. Without their participation this study would not have been possible. Researchers would also like to thank the University of Namibia for granting permission to conduct this study.

\section{Authors' Contributions}

Victoria Ben, Daniel Opotamutale Ashipala and Nestor Tomas were involved in the conceptualisation, literature review, data collection, analysis, writing of the manuscript in this study. Both authors wrote the original manuscript and approved the final manuscript for publication.

\section{Funding}

This research received no specific grant from any funding agency from the public, commercial and/or not-for profit sectors.

\section{Competing Interests Statement}

The researchers declare that they had no financial or personal relationship(s) which may have influenced them inappropriately in the writing of this article.

\section{References}

Brink, H., van der Walt, C., \& van Rensburg, G. (2018). Fundamentals of Research Methodology for Healthcare Professionals. Cape Town, South Africa: Juta and Company Ltd.

Babbie, E., \& Mouton, J. (2016). The Practise of Social Research. Cape Town: Oxford University Press.

Belachew Fekede, B. (2015). Assessing Student Awareness towards the Effects of Unintended Pregnancy: The case of Female students' at Mettu College of Teachers Education. Research on Humanities and Social Sciences, 5(1).

Burns, N., \& Groove, S. K. (2013). Understanding Nursing Research (8th ed.). Philadelphia: Saunders.

Coetzee, M. H., \& Ngunyulu, R. N. (2015). Assessing the use of contraceptives by female undergraduate students in a selected higher educational institution in Gauteng. Curationis, 38(2), 1535. https://doi.org/10.4102/curationis.v38i2.1535

Conradie L. (2019). Tertiary students' experiences and needs related to unplanned pregnancies and the termination of pregnancy: practice guidelines for psychosocial support (PhD Dissertation, University of South Africa, Pretoria, South Africa).

Creswell, J. (2013). Qualitative inquiry \& research design: Choosing among five approaches (3rd ed.). Los Angeles: SAGE Publications.

De Vos, A. S., Strydom, H., Fouche, C. B., \& Delport, C. S. L. (2011). Research at grassroots for the social sciences and human service professional (3rd ed.). Pretoria: Van Schaik.

Dick, B., \& Ferguson, B. J. (2015). Health for the worlds adolescents: a second chance in the second decade. $J$ Adolesc Health, 56(1), 3-6. https://doi.org/10.1016/j.jadohealth.2014.10.260

Donatus, L., Sama, D. J., Tsoka-Gwegweni. J. M., \& Cumber, S. N. (2018). Factors associated with adolescent school girl's pregnancy in Kumbo East Health District North West region Cameroon. Pan African Medical Journal (open access). https://doi.org/10.11604/pamj.2018.31.138.16888

Fekende, B. (2015). Assessing Student Awareness towards the Effects of Unintended Pregnancy: The case of Female students' at Mettu College of Teachers Education.

Grove, S. K., Burns, N., \& Gray, J. (2013). The practice of nursing research: Appraisal, synthesis, and generationof evidence (7th ed.). Missouri: Elsevier Ltd.

General Household Survey. (2017). Statistics South Africa. Pretoria, South Africa. Retrieved 1 November, 2020, from www.statssa.gov.za

Krugu, J. K., Mevissen, F., \& Ruiter, R. (2017). Beyond love: a qualitative analysis of factors associated with teenage pregnancy among young women with pregnancy experience in Bolgatanga, Ghana. Culture Health \& Sexuality, 19(3), 293-307. https://doi.org/10.1080/13691058.2016.1216167

Legal Assistance Centre. (2017). Namibia Gender Analysis. Retrieved from https://eeas.europa.eu/sites/eeas/files/namibia_gender_analysis_2017.pdf 
Leedy, P. D., \& Ormrod, J. E. (2013). Practical research: Planning and design (8th ed.). New Jersey: Pearson Prentice-Hall.

Lincoln, Y. S., \& Guba, E. (1985). Naturalistic inquiry. London: SAGE Publications. https://doi.org/10.1016/0147-1767(85)90062-8

Mathews, S., \& Mekuria, A. (2018). Teenage Pregnancy and Its Associated Factors among School Adolescents of Arba Minch Town, Southern Ethiopia. Ethiopian journal of health sciences, 28(3), 287-298. https://doi.org/10.4314/ejhs.v28i3.6

Maree, K. (2016). First steps in research. Pretoria: Van Schaik.

Ministry of Health and Social Services (MoHSS). (2013). Namibia demographic Health Survey. Windhoek: Government of Republic of Namibia.

Ministry of Health and Social Services (MoHSS). (2008). Draft Consolidated National Reproductive and Child Health Policy. Windhoek: Government of Republic of Namibia.

Mutinta G, Govender K, George, G \& Gow J. (2014). The influence of biological factors on students' sexual behaviour at the University of KwaZulu-Natal, South Africa. African Journal of AIDS Research, 13(4). https://doi.org/10.2989/16085906.2014.961938

Mutsindikwa, T., Ashipala, D. O., Endjala, T., \& Tomas, N. (2019). Knowledge, Attitudes and Practices of Contraception among tertiary students at the University Campus in Namibia. Global Journal of Health Sciences (GJHS). https://doi.org/10.5539/gjhs.v11n6p180

Polit, D. F., \& Beck, C. T. (2017). Nursing Research: Generating and Assessing Evidence for Nursing Practice WoltersKluwer (10th ed.). Philadelphia: Lippincott Williams Watkins.

Qolesa, K. S. (2017). Factors influencing teenage pregnancy in Heidedal location, Mangaung district. Master in Public Health. University of Western Cape.

Sinead, M. C., Cook, S. M. C., \& Cameron, S. T. (2015). Social issues of teenage pregnancy. Obstetrics, Gynaecology and Reproductive Medicine, 25(9), 243-248. https://doi.org/10.1016/j.ogrm.2015.06.001

Tladi, F. M., \& Jali, N. M. (2014). Factors contributing to pregnancy amongst female students at the University of Limpopo, Turfloop campus, South Africa. African Journal of Physical, Health Education, Recreation and Dance, June(Supplement1:2), 275-283.

Thongnopakun, S., Pumpaibool, T., \& Somrongthong, R. (2018). The association of sociodemographic characteristics and sexual risk behaviors with health literacy toward behaviors for preventing unintended pregnancy among university students. Journal of multidisciplinary healthcare, 11, 149-156. https://doi.org/10.2147/JMDH.S156264

Tshomo, U., Dorji, S. W., Wangchuk, D., \& Tshering, K. (2018). Perceptions and Experiences of Unplanned Pregnancies among University Students: A Case study at Paro College of Education.

UNICEF. (2013). A Namibia fit for children. Retrieved from https://www.unicef.org/namibia/Adolescents_fact_sheet_print.pdf

WHO. (2018). Worldwide, an estimated 25 million unsafe abortions occur each year. Retrieved from http://www.who.int/news-room/detail/28-09-2017-worldwide-an-estimated-25-million-unsafe-abortions-occ ur-each-year

WHO. (2018). Family planning/contraception. Retrieved from http://www.who.int/news-room/fact-vedsheets/detail/family-planning-contraception

Zungu, L. I., \& Manyisa, Z. M., (2009). Factors contributing to pregnancies among student nurses at a nursing college in mpumalanga province, South Africa. Africa Journal of Nursing and Midwifery, 11(2), 61-74.

\section{Copyrights}

Copyright for this article is retained by the author(s), with first publication rights granted to the journal.

This is an open-access article distributed under the terms and conditions of the Creative Commons Attribution license (http://creativecommons.org/licenses/by/4.0/). 